\title{
Pathological Definition and Evaluation of Diabetic Neuropathy and Clinical Correlations
}

\author{
Anders A.F. Sima
}

\begin{abstract}
Diabetic neuropathy is a major complication of diabetes mellitus affecting to an equal extent type 1 and type 2 patients. Various mechanisms including metabolic and vascular abnormalities have been proposed to explain the progressive pathological changes that occur in peripheral nerve. Regardless of the precise mechanisms, structural abnormalities lead to functional changes which eventually result in the clinical manifestations of diabetic neuropathy. Possible interventions that may influence the progressive disease process include intensive insulin therapy and aldose reductase inhibitors. This article reviews the proposed pathogenetic mechanisms underlying diabetic neuropathy, their effects on nerve histomorphometric changes and ultrastructure typical of diabetic neuropathy, and potential therapeutic interventions and their impact on disease progression.
\end{abstract}

\begin{abstract}
Résumé: Définition anatomopathologique et évaluation de la neuropathie diabétique - corrélations cliniques. La neuropathie diabétique est une complication majeure du diabète atteignant aussi bien les patients ayant un diabète de type 1 que de type 2 . Différents mécanismes, dont les anomalies métaboliques et vasculaires, ont été proposés pour expliquer les changements anatomopathologiques progressifs observés dans les nerfs périphériques. Quelqu'en soit le mécanisme précis, les anomalies de structure amènent des changements fonctionnels qui éventuellement se manifestent cliniquement par la neuropathie diabétique. Il est possible que l'insulinothérapie intensive et les inhibiteurs de l'aldose réductase puissent influencer le processus pathologique. Dans cet article. nous revoyons les mécanismes pathogéniques qui ont été proposés, leurs effets sur les changements histomorphométriques et l'ultra structure typique de la neuropathie diabétique, les interventions thérapeutiques possibles et leur impact sur la progression de la maladie.
\end{abstract}

Can. J. Neurol. Sci. 1994; 21: Suppl. 4-S13-S17

Diabetic neuropathy is the most common symptomatic complication of diabetes and encompasses a group of clinical syndromes with manifestations involving somatic and autonomic peripheral nerve. The most common neuropathic syndrome is the distal symmetric polyneuropathy, believed to affect $17 \mathrm{mil}-$ lion people in the United States and Europe. Distal symmetric polyneuropathy is responsible for an enormous morbidity among diabetic patients, with consequent direct and indirect economic implications for patients and society. Diabetic neuropathy occurs with the same frequency in type 1 and type 2 diabetic subjects. ${ }^{1}$

The pathogenetic mechanisms responsible for diabetic neuropathy are not fully understood. Epidemiological and clinical data have implicated hyperglycemia and its metabolic consequences in the genesis of diabetic neuropathy. The cumulative prevalence of diabetic neuropathy increases with duration of diabetes both in type 1 and type 2 diabetes despite differences in diabetogenic processes. ${ }^{1}$

In the last decade, we have gained increasing insights into underlying metabolic disturbances that are linked to the characteristic progressive functional disturbances and structural damage affecting peripheral nerves in diabetes.
One of the most investigated pathogenetic theories in diabetic neuropathy is the hyperglycemia-polyol-myoinositol hypothesis. Polyol-pathway activation was initially suggested to underlie diabetic cataract formation in the lens. 2 Increased polyol metabolites have subsequently been demonstrated in various tissues susceptible to secondary diabetic complications, including peripheral nerve, and are believed to be the major initiating event in the pathogenesis of diabetic neuropathy.

Increased glucose levels have been shown to initiate increased gene expression of the enzyme aldose reductase, ${ }^{3}$ which activates the polyol pathway, leading to intracellular accumulation of sorbitol and fructose. Accumulation of the intracellular osmolyte sorbitol is associated with a reciprocal diminution of intracellular myoinositol, ${ }^{4}$ with subsequent adverse effects on phosphoinositide metabolism. The resulting defects particularly on $\mathrm{Na}^{+} / \mathrm{K}^{+}$ATPase activity appear to play a

From the Departments of Pathology und Internal Medicine. University of Michigan. Ann Arbor. Michigan, USA.

Reprint requests to: Anders A.F. Sima, University of Michigan Medical Center, 1331 E Ann Street, Box 0580. Ann Arbor, Michigan, USA 48109 
central role in explaining the acute slowing of nerve conduction velocity and defects in axonal transport. ${ }^{5}$ One of the most intriguing linkages between the activated polyol pathway and nerve hypoxia secondary to decreased blood flow is the possibility that aldose reductase may compete with nitric oxide synthase for the reduced form of nicotinamide-adenine dinucleotide phosphate, leading to impaired synthesis of the potent vasodilator nitric oxide. ${ }^{6}$ However, other metabolic abnormalities related to hyperglycemia are likely to have additional and/or potentiating effects on nerve function and structure in diabetic peripheral nerve.

The role of endoneurial microvascular abnormalities predisposing to peripheral nerve hypoxia and/or ischemia has been proposed as an important pathogenetic mechanism in diabetic neuropathy. The functional basis for nerve hypoxia has been demonstrated in diabetic animals, showing reduced endoneurial blood flow and oxygen tension and increased vascular resistance associated with decreased nerve conduction velocity. ${ }^{7.8}$ In short term experiments these abnormalities can be corrected by a variety of agents with vasodilatory action like prostaglandin $E_{1}$ analogues, guanethidine, and aminoguanidine. It is of interest to note that also aldose reductase inhibitors correct endoneurial blood flow, suggesting a metabolic link between the polyolpathway and vasodilatory mechanisms as mentioned above.

One of the most notable findings in diabetic neuropathy is the slowing of nerve conduction velocity. The saltatory propagation of the nerve impulse depends on the generation of a sufficient membrane potential at the node of Ranvier. Because nerve conduction slowing is an early abnormality of diabetic neuropathy, early structural abnormalities have been sought at the node of Ranvier.

A sequence of structural changes of the node was first explored in the experimental diabetic BB/W-rat. The earliest detectable structural change consists of an abnormal swelling of the nodal axon related to increased intra-axonal $\mathrm{Na}^{+}$secondary to the $\mathrm{Na}^{+} / \mathrm{K}^{+}$ATPase defect. Nodal swelling is followed by detachment of the terminal myelin loops, which delineate the node - an abnormality referred to as axoglial dysjunction (Figure 1), which results in a breach in the paranodal ion-channel barrier and eventually leads to myelin retraction from the nodal area. These neuropathological changes are not acutely reversible and are associated with the more persistent nerve conduction slowing in diabetic neuropathy. ${ }^{9}$ The anchoring of the terminal Schwann cell loops to the axolemma via axoglial junctions provides a structual barrier that prevents lateral migration of nodal Na-channels necessary for normal generation of the membrane potential and the propagation of the electrical impulse. The subsequent demyelination of the paranodal axon exposes paranodal $\mathrm{K}^{+}$channels associated with increased $\mathrm{K}^{+}$ leakage currents, which leads to further impairment of nerve conduction. This sequence of structural abnormalities occurring at the node of Ranvier (Figure 2) with associated electrophysiological phenomena was subsequently identified in human diabetic neuropathy.

Ultrastructural changes in sural nerve biopsies obtained from patients with insulin-dependent diabetes mellitus were reported on by Bischoff, who emphasized structural changes of myelinated axons that were atrophied in proportion to the myelin sheath. ${ }^{10}$ More recent studies in both humans and experimental animals have expanded on those observations, and it is now generally accepted that axonal atrophy is the main structural abnormality of diabetic neuropathy and is associated with a defect in the transportation of axonal building blocks from the nerve cell body to the axon (Figure 3). Axonal atrophy is not a static condition but heralds the degeneration of the axon, starting at the most distal part of the axon and progresses proximally. Similar

\section{Normal}

\section{Diabetes}

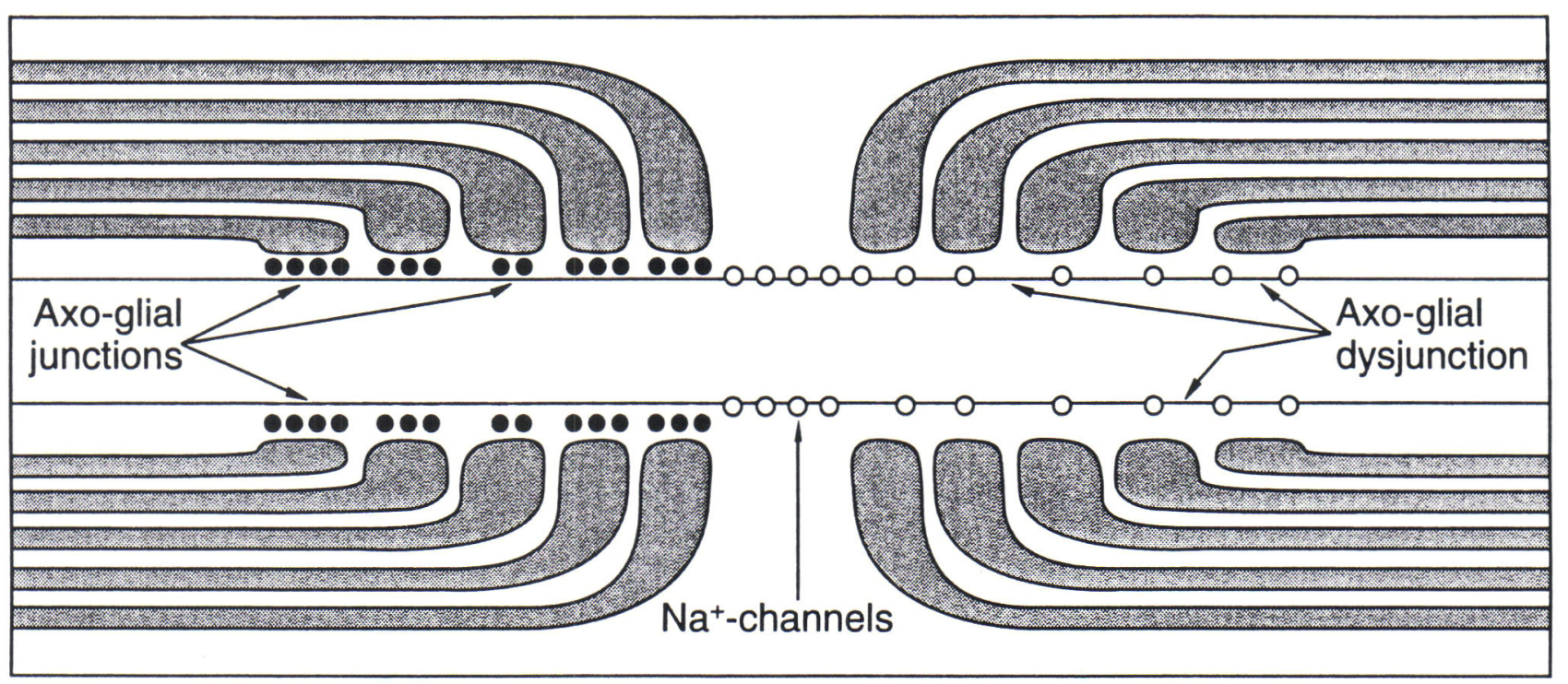

Figure 1: Schematic illustration of the normal node of Ramvier (left) where the nodal $\mathrm{Na}^{+}$-channels are confined to the nodal gap by an intact paranodal ion channel barrier. In diabetic neuropaty this barrier is damaged (axoglial dysjumtion) allowing for lateral displacement of Na+-chamels (right side of the node). 


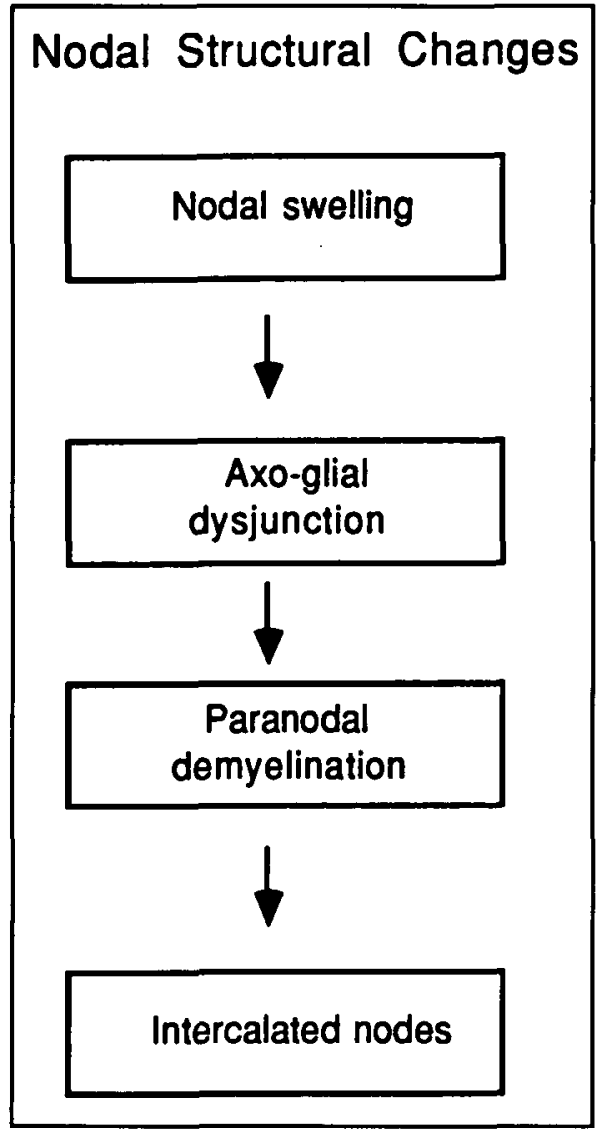

Figure 2: Sequence of quantifiable structural changes affecting the node of Ranvier. sensory axon and progress towards the cell soma, making diabetic neuropathy a truly central-peripheral axonopathy of dying back type. Axonal atrophy also affects unmyelinated peripheral nerve fibers, conducting pain and thermal sensation, and are also responsible for autonomic innervation of blood vessels, sweat glands and internal organs. The distal to proximal progression of nerve fiber degeneration explains the temporal and spatial distribution of neurological deficits which progress from the most distal parts of the limbs proximally.'

Healthy nerve fibers that have been damaged through trauma or ischemia regenerate and eventually reconnect with the target organ, which regains its function. In diabetes, regeneration of nerve fibers is impaired. The subsequent progressive net loss of functional units which coincides with the emergence of clinical symptoms, is likely the most important pathological change in the diabetic nerve, and as such forms the very definition of diabetic neuropathy. ${ }^{11,12}$

Diabetes affects not only the nerve fibers of the peripheral nerve but also the supporting tissue, such as the microvasculature. Structural changes of the endoneurial vasculature such as basement membrane thickening, endothelial cell proliferation, "closed capillaries", and platelet thrombi may explain focal and centrofascicular nerve fiber loss. Furthermore, endoneurial vessels show loss of junctional structures joining the endothelial



Figure 3: Sequence of axonal changes in diabetic neuropathy.

cells (endothelial cell dysjunction), which normally constitute the blood-nerve barrier. ${ }^{13}$ The latter change is analogous to axoglial dysjunction and may provide the basis for increased permeability of peripheral nerve capillaries in diabetes. Structural changes affecting endoneurial vessels support a vascular component in the pathogenesis of diabetic neuropathy. ${ }^{14.15}$

There is now mounting evidence that diabetic neuropathy is initiated and sustained by metabolic abnormalities. The subsequent emergence of subtle structural abnormalities, particularly of the paranodal apparatus, can be correlated with the progressive decline of the increasingly less reversible nerve conduction defect. ${ }^{16}$ These metabolic, functional and structural relationships (Figure 4) from the conceptual basis for the clinical testing and use of pharmacological compounds directed against specific metabolic pathways in the diabetic nerve, such as the polyol pathway.

Ever since nerve degeneration and nerve fiber loss were implicated in the development of diabetic neuropathy, much attention has been focused on the role of the polyol pathway and the enzyme aldose reductase. Therefore, the ability to inhibit sorbitol accumulation pharmacologically represents one therapeutic basis for the prevention and/or reversal of nerve damage associated with these metabolic alterations. It should however be mentioned that other experimental compounds, such as 


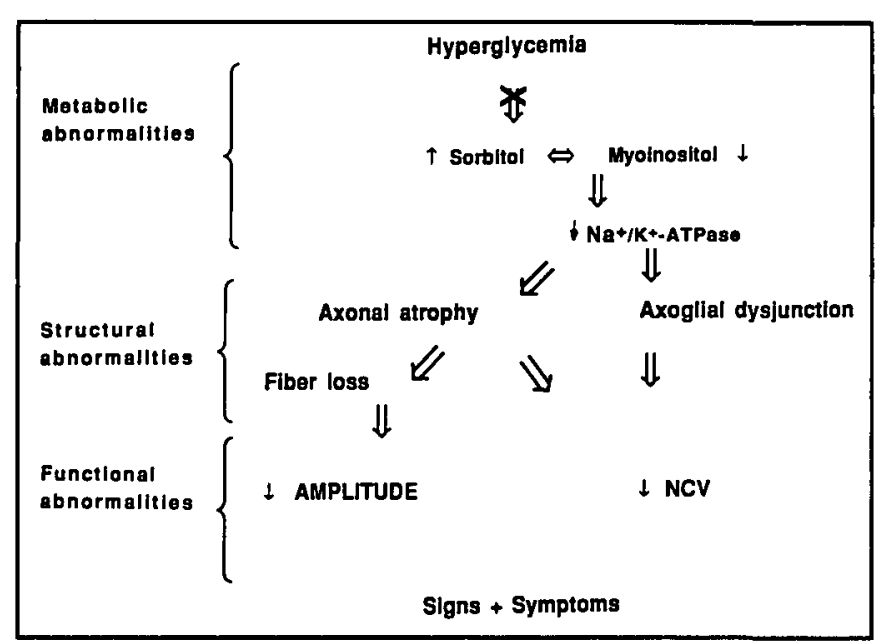

Figure 4: Interrelationships between metabolic, structural, and functional abnormalities in diabetic nerve. Inhibition of the hyperglycemia-driven polyol-pathway, indicated by a cross-out ( $X$ "), prevents subsequent metabolic, structural, and functional abnormalities.

evening primrose oil and acetyl-L-carnitine, the actions of which are less well defined, have demonstrated promising results in experimental animals.

The nerve fiber loss, atrophy and injury in diabetic distal polyneuropathy and their responses to metabolic interventions can be assessed by computer-driven morphometric analysis of sural nerve structure. These techniques allow for reproducible and highly sensitive quantitative measures of a series of meaningful light microscopic and ultrastructural neuropathologic abnormalities that show highly significant correlations with electrophysiological and biochemical parameters. The application of such morphometric techniques has demonstrated that most of the structural changes that occur in diabetic neuropathy progress with the duration of the diabetes and the treatment with biologically active aldose reductase inhibitors (ARI's) not only halts the progression of this complication but may even improve the condition. ${ }^{17}$

ARI's have been shown to prevent or reverse the metabolic and even structural changes found in nerves of diabetic rat models (Figure 4). Long-term human clinical trials have recently been completed, and morphometric analysis of sural nerve biopsies have yielded some meaningful insights into the effects of ARI's on diabetic nerve fiber damage. ${ }^{17,18}$

In two clinical trials investigating the ARI-agents sorbinil and tolrestat, biochemical analysis of sural nerve biopsies revealed significant decreases in sorbitol levels in treated patients. ${ }^{17.18}$ That beneficial metabolic effect was shown to be associated with an increased regenerative capacity of the nerve, resulting in increased fiber density and therefore a partial correction of the characteristic nerve fiber loss. A significant correlation was demonstrated between the duration of ARI-treatment and the extent of nerve fiber regeneration, indicating that prolonged ARI-treatment does stimulate nerve fiber regeneration. ${ }^{18}$ A number of other lesions characteristic of diabetic neuropathy, such as axoglial dysjunction and axonal atrophy, showed morphometrically correction toward normal.

Such results would indicate that ARI's that are biologically active in the nerve do have a beneficial effect on peripheral nerve morphometric parameters in sural nerves in patients with overt diabetic polyneuropathy, arguing strongly for the role of the polyol pathway in the pathogenesis of diabetic neuropathy and the promise of ARI therapy in the treatment of this common diabetic complication.

Despite the progress that has been made during the last decade with respect to our understanding of pathogenetic mechanisms, the dynamic structural changes, and of potential treatments of this common neurological disorder, a number of questions remain to be explored. Several laboratories are currently examining the possibility of a unifying pathogenetic mechanism that would explain both the rheological and vascular changes and those affecting nerve fiber function and structure. Another unsolved problem concerns the underlying mechanism responsible for impaired nerve fiber regeneration and nerve fiber loss in diabetes, the very hallmark of the disease. Once we understand all the aspects of this disease process, we will be able to design therapies and preventive measures for this common, debilitating complication of diabetes.

\section{REFERENCES}

1. Greene DA, Sima AAF, Alberts JW and Pfeifer M. Diabetic neuropathy. In: Ellenberg and Rifkin Diabetes Mellitus. Rifkin $\mathrm{H}$ and Porte D, eds. New York. Elseviers Publishing Company, 1989: 710-755.

2. Kinoshita JH, Futterman S, Satoh K and Merola LO. Factors affecting the formation of sugar alcohols in ocular lens. Biochim Biophys Acta 1963; 74: 340-350.

3. Gharhar A, Chakrabarti S, Murphy LJ and Sima AAF. Effect of insulin and statil on aldose reductase expression in diabetic rats. Diabetes 1991; 40: 1391-1396.

4. Stevens MJ, Lattimer SA, Kamijo M, et al. Osmotically induced nerve taurine depletion in experimental diabetes: a hypothetical mediator of painful neuropathy. Diabetologia 1993; 36: 608-614.

5. Greene DA, Lattimer SA and Sima AAF. Sorbitol, myo-inositol and sodium-potassium ATPase in the pathogenesis of diabetic complications. N Engl J Med 1987; 316: 599-606.

6. Greene DA, Sima AAF, Stevens M, et al. Aldose reductase inhibitors: an approach to the treatment of the nerve damage of diabetic neuropathy. Diabetes Metabol Rev 1993; 9: 189-217.

7. Low PA, Lagerlund TD and McManis PG. Nerve blood flow and oxygen delivery in normal, diabetic and ischemic neuropathy. Int Rev Neurobiol 1989; 31: 355-438.

8. Cameron NE, Cotter MA, Ferguson K, Robertson S and Radcliffe MA. Effects of chronic alpha-adrenergic receptor blockade on peripheral nerve conduction, hypoxic resistance, polyols, $\mathrm{Na} / \mathrm{K}$ ATPase activity, and vascular supply in STZ-D rats. Diabetes 1991; 40: 1652-1658.

9. Sima AAF, Lattimer SA, Yagihashi S and Greene DA. "Axo-glial dysjunction": a novel structural lesion that accounts for poorly reversible slowing of nerve conduction in the spontaneously diabetic BB-rat. J Clin Invest 1986; 77: 474-484.

10. Bischoff A. Diabetische Neuropathie. Dtsch Med Wochenschr 1968; 93: 237-241.

11. Concensus Statement. Proceedings of a Consensus Development Conference on Standardized Measures in Diabetic Neuropathy. Neurology 1992; 42: 1823-1839.

12. Dyck PJ, Karnes J and O'Brien PC. Diagnosis, staging, and classification of diabetic neuropathy and associations with other complications. In: Diabetic Neuropathy. Dyck PJ, Thomas PK, Asbury AK, Winegrad AI and Porte D, eds. Philadelphia, WB Saunders, 1987; 36-44.

13. Sima AAF, Nathaniel V, Prashar A, Bril V and Greene DA. Endoneurial microvessels in human diabetic neuropathy: endothelial cell dysjunction and lack of treatment effect by aldose reductase inhibitor. Diabetes 1991; 40: 1090- 1099. 
14. Dyck PJ, Karnes JL, $\mathrm{O}^{\circ}$ Brien $\mathrm{P}$, et al. The spatial distribution of fiber loss of diabetic polyneuropathy suggests ischemia. Ann Neurol 1986; 19: 440-449.

15. Dyck PJ. Hypoxic neuropathy: does hypoxia play a role in diabetic neuropathy? Neurology 1989; 39: 111-118.

16. Sima AAF, Prashar A, Nathaniel V, et al. Overt diabetic neuropathy: repair of axo-glial dysjunction and axonal atrophy by aldose reductase inhibition and its correlation to improvement in nerve conduction velocity. Diabetic Medicine 1993; 10: 115-121.
17. Sima AAF, Bril V. Nathaniel V, McEwen TA, et al. Regeneration and repair of myelinated fibers in sural nerve biopsies from patients with diabetic neuropathy treated with an aldose reductase inhibitor. N Eng J Med 1988; 319: 548-555.

18. Sima AAF, Greene DA, Brown MB, et al. and the Tolrestat Study Group. Effect of hyperglycemia and the aldose reductase inhibitor tolrestat on sural nerve biochemistry and morphometry in advanced diabetic peripheral polyneuropathy. J Diab Complications 1993; 7: 157-169. 